\title{
Averaging principle for fractional heat equations driven by stochastic measures ${ }^{\star}$
}

\author{
Guangjun Shen ${ }^{\mathrm{a}}$, Jiang-Lun $\mathrm{Wu}^{\mathrm{b}, *}$, Xiuwei Yin ${ }^{\mathrm{a}}$ \\ ${ }^{a}$ Department of Statistics, Anhui Normal University, Wuhu 241000, China. \\ ${ }^{b}$ Department of Mathematics, Swansea University, Swansea, SA1 8EN, UK.
}

\begin{abstract}
In this paper, by utilising Besov space techniques, we establish the time averaging principle for a heat equation with fractional Laplace driven by a general stochastic measure $\mu$ which is assumed (only) to satisfy the $\sigma$-additivity in probability.
\end{abstract}

Keywords: Averaging principle; fractional heat equation; stochastic measure; Besov space.

2010 MSC: 60H15, 60G57.

\section{Introduction}

Averaging method is an important tool for investigations of nonlinear dynamical systems. It helps us to obtain approximate solutions to differential equations in mechanics, mathematics, physics and many other subjects.

The theory of averaging principle for the deterministic systems was first appeared in [1]. Then, Khasminskii in his seminal paper 4. studied the averaging principle for stochastic differential equations. Since then, it becomes an active research topic in the study of stochastic dynamic systems, and the averaging principle was investigated for many different types of equations, see, for example, [14, 15, 8, 6, 2, 12, 7, 13, and the references therein.

Recently, in [9, Vadym Radchenko investigated a class of stochastic heat equations driven by a stochastic measure $\mu$, wherein the stochastic measure $\mu$ only satisfies the $\sigma$-additivity in probability (see the precise description in Section 2). Moreover, the same author established in [10] an averaging principle for such equations.

Motivated by the above results, in this short paper, we want to consider an averaging principle for the following equation

$$
\left\{\begin{array}{l}
\partial_{t} u^{\varepsilon}(t, x)=-(-\Delta)^{\frac{\beta}{2}} u^{\varepsilon}(t, x)+f\left(u^{\varepsilon}(t, x)\right)+\sigma(t / \varepsilon, x) d \mu(x), t \in(0, T], x \in \mathbb{R}, \\
u^{\varepsilon}(0, x)=u_{0}(x),
\end{array}\right.
$$

for arbitrarily given $T>0$, where $\varepsilon>0,1<\beta \leq 2$, the both coefficients $f: \mathbb{R} \rightarrow \mathbb{R}$ and $\sigma: \mathbb{R}_{+} \times \mathbb{R} \rightarrow \mathbb{R}$, as well as the initial data $u_{0}(x)=u_{0}(x, \omega): \mathbb{R} \times \Omega \rightarrow \mathbb{R}$ are bounded and

\footnotetext{
${ }^{\star}$ The Project-sponsored by NSFC (11901005).

${ }^{*}$ Corresponding author

Email addresses: gjshen@163.com (Guangjun Shen), j.1.wu@swansea.ac.uk (Jiang-Lun Wu), xweiyin@163.com (Xiuwei Yin)
} 
measurable with further conditions being specified in the sequel, $\mu$ is a stochastic measure defined on $\mathcal{B}(\mathbb{R})$ (see Section 2 for more details). We will make the following assumptions.

Assumption A1. There exists a constant $L_{f}>0$ such that

$$
\left|f\left(x_{1}\right)-f\left(x_{2}\right)\right| \leq L_{f}\left|x_{1}-x_{2}\right|, \forall x_{1}, x_{2} \in \mathbb{R} .
$$

Assumption A2. There exists a constant $L_{\sigma}>0$ such that for arbitrarily fixed $t \in[0, \infty)$

$$
\left|\sigma\left(t, x_{1}\right)-\sigma\left(t, x_{2}\right)\right| \leq L_{\sigma}\left|x_{1}-x_{2}\right|^{\epsilon}, \frac{1}{2}<\epsilon<1, \forall x_{1}, x_{2} \in \mathbb{R}
$$

Assumption A3. The function $\bar{\sigma}: \mathbb{R} \rightarrow \mathbb{R}$ defined via

$$
\bar{\sigma}(y):=\lim _{t \rightarrow \infty} \frac{1}{t} \int_{0}^{t} \sigma(s, y) d s, \forall y \in \mathbb{R}
$$

exists, and the function $\widetilde{\sigma}:[0, \infty) \times \mathbb{R} \rightarrow \mathbb{R}$ defined via

$$
\widetilde{\sigma}(r, y):=\int_{0}^{r}(\sigma(s, y)-\bar{\sigma}(y)) d s, r \geq 0, y \in \mathbb{R}
$$

is bounded.

Assumption A4. For $\mu$, it is required that $y \in \mathbb{R} \mapsto|y|^{\tau} \in[0, \infty)$ is integrable with respect to $\mu$ for some $\tau>\frac{1}{2}$.

Assumption A5. There exists positive random variable $C: \Omega \rightarrow(0, K]$ with $K>0$ such that

$$
\left|u_{0}(x, \omega)\right| \leq C(\omega), \quad\left|u_{0}\left(x_{1}, \omega\right)-u_{0}\left(x_{2}, \omega\right)\right| \leq C(\omega)\left|x_{1}-x_{2}\right|^{\beta\left(u_{0}\right)}, \beta\left(u_{0}\right) \geq \frac{1}{6}
$$

for $x, x_{1}, x_{2} \in \mathbb{R}$ and $\omega \in \Omega$.

Before proceeding further, we would like to point out that Assumption A3 above is rather technical, which is required by the method we used in the present paper. Nevertheless, we would like to give an example here. Assume in addition to Assumption A2, the coefficient $\sigma:(0, \infty) \times \mathbb{R} \rightarrow \mathbb{R}$ is periodic with respect to the time variable $t$, for example, $\sigma(t, x)=x^{\epsilon}(1+\cos (t))$, then Assumption A3 above clearly holds.

Similar to [9, one can show that under Assumptions $A 1 \sim A 5$, Equation (1.1) has a unique (mild) solution

$$
\begin{aligned}
u^{\varepsilon}(t, x)=\int_{\mathbb{R}} G(t, x-y) u_{0}(y) d y+\int_{0}^{t} \int_{\mathbb{R}} G(t-s, x-y) f\left(u^{\varepsilon}(s, y)\right) d y d s \\
+\int_{0}^{t} \int_{\mathbb{R}} G(t-s, x-y) \sigma\left(s / \varepsilon, u^{\varepsilon}(s, y)\right) d \mu d s,
\end{aligned}
$$

where $G(\cdot, \cdot)$ stands for the Green function associated to the following equation

$$
\left\{\begin{array}{l}
\partial_{t} u(t, x)=-(-\Delta)^{\frac{\beta}{2}} u(t, x), t \in[0, T], x \in \mathbb{R}, \\
u(0, x)=\delta_{0}(x)
\end{array}\right.
$$


In the same manner, it is also possible to deduce that under our assumptions $A 1 \sim A 5$,

$$
\left\{\begin{array}{l}
\partial_{t} \bar{u}(t, x)=-(-\Delta)^{\frac{\beta}{2}} \bar{u}(t, x)+f(\bar{u}(t, x))+\bar{\sigma}(x) d \mu(x), t \in(0, T], x \in \mathbb{R}, \\
\bar{u}(0, x)=u_{0}(x)
\end{array}\right.
$$

has a unique solution

$$
\begin{aligned}
\bar{u}(t, x)= & \int_{\mathbb{R}} G(t, x-y) u_{0}(y) d y+\int_{0}^{t} \int_{\mathbb{R}} G(t-s, x-y) f(\bar{u}(s, y)) d y d s \\
& +\int_{0}^{t} \int_{\mathbb{R}} G(t-s, x-y) \bar{\sigma}(y) d \mu d s .
\end{aligned}
$$

We aim to study the convergence of $u^{\varepsilon}(t, x) \rightarrow \bar{u}(t, x)$ as $\varepsilon \rightarrow 0$.

The rest of this paper is organised as follows. In Section 2, we introduce the stochastic measure $\mu$ and give its basic properties. In Section 3, we formulate our main result and give the proof of it. We end our paper with an appendix showing a critical lemma which is our section 4.

\section{Preliminaries}

In this section, we briefly recall the definition of stochastic measure and Besov space. Let $(\Omega, \mathcal{F}, \mathbb{P})$ be a complete probability space and $L^{0}=L^{0}(\Omega, \mathcal{F}, \mathbb{P})$ be the totality of real-valued random variables on the complete probability space $(\Omega, \mathcal{F}, \mathbb{P})$. Convergence in $L^{0}$ means the convergence in probability. Recall that $\mathcal{B}(\mathbb{R})$ stands for the Borel $\sigma$-algebra on $\mathbb{R}$.

Definition 2.1. ([9, 10]) A mapping $\mu: \mathcal{B}(\mathbb{R}) \rightarrow L^{0}$ is called a stochastic measure if it is $\sigma$ additive.

The class of stochastic measures is in fact very rich. As pointed out in [9, 10, a typical example is that $\mu$ is determined by a square integrable martingale $M_{t}$ in the manner $\mu(A)=\int_{0}^{T} 1_{A}(t) d M_{t}$ for $A \in \mathcal{B}(\mathbb{R})$, and further examples can be made from fractional Brownian motion with Hurst index $H \geq \frac{1}{2}$ and from any $\alpha$-stable random measure on $\mathcal{B}(\mathbb{R})$. Here we give another example. Let

$$
F_{t, x}(\omega)=W_{t, x}(\omega)+\int_{U_{0}} c_{1}(t, x ; z) M_{t, x}(d z, \omega)+\int_{U \backslash U_{0}} c_{2}(t, x ; z) N_{t, x}(d z, \omega)
$$

be a Lévy space-time white noise introduced in [3] (see Section 2.2 therein), then one has that

$$
\mu_{1}(A)=\int_{0}^{T} \int_{\mathbb{R}} g(t, x) 1_{A}(t) d F_{t, x}(\omega), \quad A \in \mathcal{B}(\mathbb{R})
$$

and

$$
\mu_{2}(A)=\int_{0}^{T} \int_{\mathbb{R}} g(t, x) 1_{A}(x) d F_{t, x}(\omega), \quad A \in \mathcal{B}(\mathbb{R})
$$

are both stochastic measures, provided that $g:(0, \infty) \times \mathbb{R} \rightarrow \mathbb{R}$ is bounded and progressively measurable. From this point, one can see clearly that the fractional heat equations driven by stochastic measures we are concerned in this paper cover the corresponding equations driven by all usual treatable noises in the literature. 
Assume that $f: \mathbb{R} \rightarrow \mathbb{R}$ is a measurable function, one can define an integral $\int_{A} f(x) \mu(d x), A \in$ $\mathcal{B}(\mathbb{R})$. It is clear that the integral $\int_{A} f(x) \mu(d x)$ is well defined for every bounded $f$, see $[5]$ for more details.

Consider the Besov space $B_{22}^{\alpha}([c, d])$ for any given interval $[c, d] \subset \mathbb{R}, 0<\alpha<1$, recall that the norm in this space can be defined by

$$
\|g\|_{B_{22}^{\alpha}([c, d])}:=\|g\|_{L^{2}([c, d])}+\left(\int_{0}^{d-c}\left(w_{2}(g, r)\right)^{2} r^{-1-2 \alpha} d r\right)^{\frac{1}{2}},
$$

where

$$
w_{2}(g, r):=\sup _{0 \leq h \leq r}\left(\int_{c}^{d-h}|g(y+h)-g(y)|^{2} d y\right)^{1 / 2} .
$$

For any $j \in \mathbb{Z}, n \geq 0$, put

$$
d_{k n}^{(j)}=j+k 2^{-n}, 0 \leq h \leq 2^{n}, \quad \Delta_{k n}^{(j)}=\left(d_{(k-1) n}^{(j)}, d_{k n}^{(j)}\right], 1 \leq k \leq 2^{n} .
$$

The following lemmas are important for our results (see 9 for their proofs).

Lemma 2.1. Let $\phi_{l}: \mathbb{R} \rightarrow \mathbb{R}, l \geq 1$, be measurable functions such that $\widetilde{\phi}(x)=\sum_{l=1}^{\infty}\left|\phi_{l}(x)\right|$ is integrable with respect to $\mu$. Then $\sum_{l=1}^{\infty}\left(\int_{\mathbb{R}} \phi_{l}(x) d \mu\right)^{2}<+\infty$, a.s.

Lemma 2.2. Let $Z$ be an arbitrary (nonempty) set, and $q(z, s): Z \times[j, j+1] \rightarrow \mathbb{R}$ be such that all paths $q(z, \cdot), z \in Z$, are continuous. Denote

$$
q_{n}(z, s):=q(z, j) 1_{\{j\}}(s)+\sum_{1 \leq k \leq 2^{n}} q\left(z, d_{(k-1) n}^{(j)}\right) 1_{\Delta_{k n}^{(j)}}(s) .
$$

Then the random function $\eta(z):=\int_{(j, j+1]} q(z, s) d \mu(s), z \in Z$, has a version

$$
\widetilde{\eta}(z)=\int_{(j, j+1]} q_{0}(z, s) d \mu(s)+\sum_{n \geq 1}\left(\int_{(j, j+1]} q_{n}(z, s) d \mu(s)-\int_{(j, j+1]} q_{n-1}(z, s) d \mu(s)\right) .
$$

For simplicity, we will use $C$ and $C(\omega)$ to denote finite positive constants and finite positive random constants separately whose values may be different in different positions.

\section{Main result}

Lemma 3.1. Assume that Assumption $A 1 \sim A 3$ hold, then

$$
\sup _{y \in \mathbb{R}, x>0, \varepsilon>0, t \in[0, T]}\left|\left(\frac{1}{\varepsilon}\right)^{\frac{\beta-1}{\beta}} \int_{0}^{t} G(t-s, x)[\sigma(s / \varepsilon, y)-\bar{\sigma}(y)] d s\right|<+\infty .
$$

Proof. It follows from the self-similarity property of $G(\cdot, \cdot)$ (see, for example, [11) that

$$
\begin{aligned}
& \left(\frac{1}{\varepsilon}\right)^{\frac{\beta-1}{\beta}} \int_{0}^{t} G(t-s, x)[\sigma(s / \varepsilon, y)-\bar{\sigma}(y)] d s \\
& =\left(\frac{1}{\varepsilon}\right)^{\frac{-1}{\beta}} \int_{0}^{t / \varepsilon} G(s v, x)[\sigma(t / \varepsilon-v, y)-\bar{\sigma}(y)] d v \\
& =\int_{0}^{t / \varepsilon}\left(\frac{1}{v}\right)^{\frac{1}{\beta}} G\left(1,(\varepsilon v)^{-1 / \beta} x\right)[\sigma(t / \varepsilon-v, y)-\bar{\sigma}(y)] d v \\
& =: \mathrm{I}_{1}+\mathrm{I}_{2} .
\end{aligned}
$$


On one hand, for the term $\mathrm{I}_{1}$, we have $|\sigma(s, y)-\bar{\sigma}(y)| \leq C_{\sigma}$, and observe further that $G(t, x)$ satisfies the following inequality

$$
\frac{c_{1} t}{\left(t^{1 / \beta}+|x|\right)^{1+\beta}} \leq G(t, x) \leq \frac{c_{2} t}{\left(t^{1 / \beta}+|x|\right)^{1+\beta}}, c_{1}, c_{2}>0 .
$$

Thus, it follows that

$$
\begin{aligned}
\mathrm{I}_{1} & =\int_{0}^{1}\left(\frac{1}{v}\right)^{\frac{1}{\beta}} G\left(1,(\varepsilon v)^{-1 / \beta} x\right)[\sigma(t / \varepsilon-v, y)-\bar{\sigma}(y)] d v \\
& \leq c_{2} C_{\sigma} \int_{0}^{1}\left(\frac{1}{v}\right)^{\frac{1}{\beta}} \frac{1}{\left(1+(\varepsilon v)^{-1 / \beta}|x|\right)^{2+\beta}} d v \\
& \leq c_{2} C_{\sigma} \int_{0}^{1}\left(\frac{1}{v}\right)^{\frac{1}{\beta}} d v \leq C .
\end{aligned}
$$

On the other hand, for the term $\mathrm{I}_{2}$, having that $\left|F_{\varepsilon}(r):=\int_{0}^{r}[\sigma(t / \varepsilon-v, y)-\bar{\sigma}(y)] d v\right| \leq C_{F}$, we then get

$$
\begin{aligned}
\mathrm{I}_{2}= & \int_{1}^{t / \varepsilon} v^{-\frac{1}{\beta}} G\left(1,(\varepsilon v)^{-1 / \beta} x\right)[\sigma(t / \varepsilon-v, y)-\bar{\sigma}(y)] d v \\
= & \int_{1}^{t / \varepsilon} v^{-\frac{1}{\beta}} G\left(1,(\varepsilon v)^{-1 / \beta} x\right) d F_{\varepsilon}(v) \\
= & \left.F_{\varepsilon}(v) v^{-\frac{1}{\beta}} G\left(1,(\varepsilon v)^{-1 / \beta} x\right)\right|_{1} ^{t / \varepsilon} \\
& +\int_{1}^{t / \varepsilon} F_{\varepsilon}(v) \frac{1}{\beta} v^{-\frac{1+\beta}{\beta}}\left[G\left(1,(\varepsilon v)^{-1 / \beta} x\right)+(\varepsilon v)^{-\frac{1}{\beta}} x \frac{\partial}{\partial x} G\left(1,(\varepsilon v)^{-\frac{1}{\beta}} x\right)\right] d v \\
\leq & 2 c_{2} C_{F}+\frac{C_{F}}{\beta}\left[c_{2} \int_{1}^{\infty} v^{-\frac{1+\beta}{\beta}} d v+C \int_{1}^{\infty} \frac{\left|(\varepsilon v)^{-\frac{1}{\beta}} x\right|^{\beta+1} v^{-\frac{1+\beta}{\beta}}}{\left(1+\left|(\varepsilon v)^{-\frac{1}{\beta}} x\right|^{\beta+1}\right)^{2}} d v\right] \\
\leq & 2 c_{2} C_{F}+\frac{C_{F}}{\beta}\left(c_{2}+2 C\right) \int_{1}^{\infty} v^{-\frac{1+\beta}{\beta}} d v \leq C .
\end{aligned}
$$

This completes the proof.

Our main result of the paper is the following

Theorem 3.1. Under Assumptions $A 1 \sim A 5$, there exist versions of $u^{\varepsilon}$ and $\bar{u}$ such that for any $\gamma_{1}<\frac{\beta-1}{\beta}\left(1-\frac{1}{2[\epsilon \wedge(\beta-1)]}\right)$, we have

$$
\sup _{\varepsilon>0, t \in[0, T], x \in \mathbb{R}} \varepsilon^{-\gamma_{1}}\left|u^{\varepsilon}(t, x)-\bar{u}(t, x)\right|<+\infty, \text { a.s. }
$$

Proof. We take the versions of stochastic integrals defined by Lemma 2.2 and continuous versions of $u^{\varepsilon}$ and $\bar{u}$, respectively. Let

$$
\eta_{\varepsilon}:=\int_{\mathbb{R}} d \mu(y) \int_{0}^{t} G(t-s, x-y) \sigma(s / \varepsilon, y) d s-\int_{\mathbb{R}} d \mu(y) \int_{0}^{t} G(t-s, x-y) \bar{\sigma}(y) d s .
$$

We want to show that

$$
\left|\eta_{\varepsilon}\right| \leq C(\omega) \varepsilon^{\gamma}, \text { a.s. }
$$

To this end, we denote $z:=(t, x)$, and

$$
g(z, y):=\int_{0}^{t} G(t-s, x-y)(\sigma(s / \varepsilon, y)-\bar{\sigma}(y)) d s .
$$


Then,

$$
\eta_{\varepsilon}=\int_{\mathbb{R}} g(z, y) d \mu(y)=\sum_{j \in \mathbb{Z}} \int_{(j, j+1]} g(z, y) d \mu(y) .
$$

First of all, we shall use the Besov norm to estimate $\int_{(j, j+1]} g(z, y) d \mu(y)$. Consider

$$
\begin{aligned}
& g(z, y+h)-g(z, y) \\
& =\int_{0}^{t} G(t-s, x-y)[\sigma(s / \varepsilon, y+h)-\sigma(s / \varepsilon, y)-\bar{\sigma}(y+h)+\bar{\sigma}(y)] d s \\
& \quad+\int_{0}^{t}(G(t-s, x-y-h)-G(t-s, x-y))(\sigma(s / \varepsilon, y)-\bar{\sigma}(y)) d s \\
& =: \mathrm{II}_{1}+\mathrm{II}_{2} .
\end{aligned}
$$

For the term $\mathrm{II}_{1}$, using Assumption $A 2$, we get

$$
\begin{aligned}
\mathrm{II}_{1} & \leq 2 L_{\sigma} h^{\epsilon} \int_{0}^{t} G(t-s, x-y) d s \\
& \leq 2 c_{2} L_{\sigma} h^{\epsilon} \int_{0}^{t} \frac{t-s}{\left((t-s)^{1 / \beta}+|x-y|\right)^{1+\beta}} d s \\
& \leq 2 c_{2} L_{\sigma} h^{\epsilon} \int_{0}^{t}(t-s)^{-\frac{1}{\beta}} d s \leq C h^{\epsilon} .
\end{aligned}
$$

For the term $\mathrm{II}_{2}$, note that the coefficient $\sigma$ is uniformly bounded, thus it follows further from 11 , Lemma 2.1] that

$$
\begin{aligned}
\left|\mathrm{II}_{2}\right| & \leq C \int_{0}^{t}|G(t-s, x-y-h)-G(t-s, x-y)| d s \\
& \leq C \int_{0}^{t} d s\left|\int_{x-y-h}^{x-y} \frac{\partial}{\partial z} G(t-s, z) d z\right| \\
& \leq C \int_{0}^{t} d s \int_{x-y-h}^{x-y} \frac{(t-s)|z|}{\left((t-s)^{1 / \beta}+|z|\right)^{3+\beta}} d z \\
& =C \int_{x-y-h}^{x-y} \int_{0}^{t} \frac{s|z|}{\left(s^{1 / \beta}+|z|\right)^{3+\beta}} d s d z .
\end{aligned}
$$

In order to estimate the above integration, letting $s^{1 / \beta}=r|z|$, then we have

$$
\begin{aligned}
\int_{0}^{t} \frac{s|z|}{\left(s^{1 / \beta}+|z|\right)^{3+\beta}} d s & =|z|^{\beta-2} \int_{0}^{\frac{t^{1 / \beta}}{|z|}} \frac{r^{2 \beta-1}}{(1+r)^{3+\beta}} d r \\
& \leq|z|^{\beta-2} \int_{0}^{\infty} \frac{r^{2 \beta-1}}{(1+r)^{3+\beta}} d r \\
& \leq|z|^{\beta-2}\left[\int_{0}^{1} r^{2 \beta-1} d r+\int_{1}^{\infty} r^{\beta-4} d r\right] \\
& \leq C|z|^{\beta-2} .
\end{aligned}
$$

Thus, it follows from Lemma 4.1 in the appendix that

$$
\left|\mathrm{II}_{2}\right| \leq C \int_{x-y-h}^{x-y}|z|^{\beta-2} d z \leq C h^{\beta-1} .
$$

We have therefore proved that

$$
\begin{aligned}
\left(w_{2}(g, r)\right)^{2} & \leq 2 \sup _{0 \leq h \leq r}\left[\int_{j}^{j+1-h} \mathrm{II}_{1}^{2} d y+\int_{j}^{j+1-h} \mathrm{II}_{2}^{2} d y\right] \\
& \leq C h^{2[\epsilon \wedge(\beta-1)]} .
\end{aligned}
$$


Furthermore, by Lemma 3.1, we have

$$
|g(z, y+h)|+|g(z, y)| \leq C \varepsilon^{\frac{\beta-1}{\beta}}
$$

Therefore,

$$
\left(w_{2}(g, r)\right)^{2} \leq \varepsilon^{\frac{2(\beta-1)}{\beta}}
$$

Combining (3.2) with 3.4), one then gets that

$$
\left(w_{2}(g, r)\right)^{2} \leq C r^{2[\epsilon \wedge(\beta-1)](1-\vartheta)} \varepsilon^{\frac{2(\beta-1)}{\beta} \vartheta}, 0<\vartheta<1 .
$$

Thus, for the finiteness of $\|g\|_{B_{22}^{\alpha}([j, j+1])}$, it suffices that $\vartheta<\frac{[\epsilon \wedge(\beta-1)]-\alpha}{[\epsilon \wedge(\beta-1)]}$. Let $\alpha \rightarrow 1 / 2+$, we can get $\vartheta \rightarrow\left(1-\frac{1}{2[\epsilon \wedge(\beta-1)]}\right)-$.

It follows from 3.2 that $|g(z, j)| \leq C \varepsilon^{\gamma_{1}}$ and $\|g(z, \cdot)\|_{L^{2}([j, j+1])} \leq C \varepsilon^{\gamma_{1}}$ hold for $\gamma_{1}<\frac{\beta-1}{\beta}$. Consequently, for any $\gamma_{1}<\frac{\beta-1}{\beta}\left(1-\frac{1}{2[\epsilon \wedge(\beta-1)]}\right)$, there exists $\alpha>1 / 2$, such that

$$
\|g(z, \cdot)\|_{B_{22}^{\alpha}([j, j+1])} \leq C \varepsilon^{\gamma_{1}} .
$$

Using Cauchy-Schwarz inequality, we further get

$$
\begin{aligned}
\left|\eta_{\varepsilon}\right| \leq & \sum_{j \in \mathbb{Z}}\left|\int_{(j, j+1]} g(z, y) d \mu(y)\right| \leq \sum_{j \in \mathbb{Z}}|g(z, j) \mu((j, j+1])| \\
& +C \sum_{j \in \mathbb{Z}}\|g(z, \cdot)\|_{B_{22}^{\alpha}([j, j+1])}\left\{\sum_{n \geq 1} 2^{n(1-2 \alpha)} \sum_{1 \leq k \leq 2^{n}}\left|\mu\left(\Delta_{k n}^{(j)}\right)\right|^{2}\right\}^{1 / 2} \\
\leq & C \varepsilon^{\gamma_{1}}\left[\sum_{j \in \mathbb{Z}}|\mu((j, j+1])|+\sum_{j \in \mathbb{Z}}\left\{\sum_{n \geq 1} 2^{n(1-2 \alpha)} \sum_{1 \leq k \leq 2^{n}}\left|\mu\left(\Delta_{k n}^{(j)}\right)\right|^{2}\right\}^{1 / 2}\right] \\
\leq & C \varepsilon^{\gamma_{1}}\left[\left(\sum_{j \in \mathbb{Z}}(|j|+1)^{\varrho} \mu^{2}((j, j+1])\right)^{1 / 2}\left(\sum_{j \in \mathbb{Z}}(|j|+1)^{-\varrho}\right)^{1 / 2}\right. \\
& \left.\left.+\left(\sum_{j \in \mathbb{Z}} \sum_{n \geq 1} \sum_{1 \leq k \leq 2^{n}}(|j|+1)^{\varrho} 2^{n(1-2 \alpha)}\left|\mu\left(\Delta_{k n}^{(j)}\right)\right|^{2}\right)^{1 / 2}(|j|+1)^{-\varrho}\right)^{1 / 2}\right]
\end{aligned}
$$

for any $\varrho>1$. Observe that $\sum_{j \in \mathbb{Z}}(|j|+1)^{-\varrho}<+\infty$. Define

$$
\left\{\phi_{l}^{(1)}(y), l \geq 1\right\}:=\left\{(|j|+1)^{\frac{e}{2}} 1_{(j, j+1]}(y), j \in \mathbb{Z}\right\}
$$

and

$$
\left\{\phi_{l}^{(2)}(y), l \geq 1\right\}:=\left\{(|j|+1)^{\frac{\rho}{2}} 2^{\frac{n-2 \alpha n}{2}} 1_{\Delta_{(k n)}^{(j)}}(y), j \in \mathbb{Z}, n \geq 1,1 \leq k \leq 2^{n}\right\} .
$$

Then, $\sum_{l=1}^{\infty}\left|\phi_{l}^{(i)}(y)\right| \leq C\left(|y|^{\varrho / 2}+1\right), i=1,2$. Using the integrability of $|y|^{\tau}$, we get 3.1). Finally, as the same proof of Step $\mathbf{2}$ in [10, we complete the proof. 


\section{Appendix}

Lemma 4.1. Let $x, y \in \mathbb{R}, 0<h<1$ and $1<\beta \leq 2$, then there exists a positive constant $C_{\beta}>0$, such that

$$
I(h)=\int_{x-y-h}^{x-y}|z|^{\beta-2} d z \leq C_{\beta} h^{\beta-1} .
$$

Proof. We will divide our proofs into three cases.

Case I: For $x-y \leq 0$, we have

$$
I(h)=\int_{x-y-h}^{x-y}(-z)^{\beta-2} d z \leq \frac{1}{\beta-1} h^{\beta-1} .
$$

Case II: For $0<x-y \leq 2$. We get

$$
\begin{aligned}
I(h) & =\int_{x-y-h}^{x-y}\left(|z|^{\beta-2} 1_{\{2 h<x-y\}}+|z|^{\beta-2} 1_{\{2 h \geq x-y\}}\right) d z \\
& \leq \int_{x-y-h}^{x-y} z^{\beta-2} 1_{\{2 h<x-y\}} d z+\int_{x-y-h}^{x-y}|z|^{\beta-2} 1_{\{2 h \geq x-y\}} d z \\
& \leq h^{\beta-1} 1_{\{2 h<x-y\}}+\int_{x-y-h}^{x-y} z^{\beta-2} 1_{\{h \leq x-y \leq 2 h\}} d z+\int_{x-y-h}^{x-y}|z|^{\beta-2} 1_{\{x-y \leq h\}} d z \\
& \leq h^{\beta-1}+\frac{1}{\beta-1} h^{\beta-1}+\int_{0}^{h-x+y} z^{\beta-2} 1_{\{x-y \leq h\}} d z+\int_{0}^{x-y} z^{\beta-2} 1_{\{x-y \leq h\}} d z \\
& \leq h^{\beta-1}+\frac{1}{\beta-1} h^{\beta-1}+\frac{1}{\beta}(h-x+y)^{\beta-1} 1_{\{x-y \leq h\}}+\frac{1}{\beta-1}(x-y)^{\beta-1} 1_{\{x-y \leq h\}} \\
& \leq C_{\beta} h^{\beta-1} .
\end{aligned}
$$

Case III: For $x-y>2$, it is clear that $x-y-h>x-y-1>1$, so that

$$
I(h) \leq \int_{x-y-h}^{x-y} d z=h .
$$

We are done.

Acknowledgment We thank the referees for their careful reading of our manuscript and for the detailed and constructive comments, which have led to improve the presentation of the paper.

\section{References}

\section{References}

[1] N. N. Bogoliubov, Y. A. Mitropolsky, Asymptotic Methods in the Theory of Non-linear Oscillations, Gordon and Breach Science Publishers, New York, 1961.

[2] Z. Dong, X. Sun, H. Xiao, J. Zhai, Averaging principle for one dimensional stochastic Burgers equation, J. Differential Equations 265 (2018) 4749-4797.

[3] N. Jacob, A. Potrykus, J.-L. Wu, Solving a non-linear stochastic pseudo-differential equation of Burgers type, Stoch. Proc. Appl. 120 (2010) 2447-2467. 
[4] R. Z. Khasminskii, On an averging principle for Itô stochastic differential equations, Kibernetica. 4 (1968) 260-279.

[5] S. Kwapień, W. A. Woyczyński, Random Series and Stochastic Integrals: Single and Multiple. Birkhäuser, Boston, 1992.

[6] W. Liu, M. Röckner, X. Sun, Y. Xie, Averaging principle for slow-fast stochastic differential equations with time dependent locally Lipschitz coefficients, J. Differential Equations 268 (2020) 2910-2948.

[7] B. Pei, Y. Xu, J.-L. Wu, Stochastic averaging for stochastic differential equations driven by fractional Brownian motion and standard Brownian motion. Appl. Math. Lett. 100 (2020) 106006.

[8] B. Pei, Y. Xu, J.-L. Wu, Two-time-scales hyperbolic-parabolic equations driven by Poisson random measures: Existence, uniqueness and averaging principles, J. Math. Anal. Appl. 447 (2017) 243-268.

[9] V. Radchenko, Mild solution of the heat equation with a general stochastic measure. Studia Math. 194 (2009) 231-251.

[10] V. Radchenko, Averaging principle for the heat equation driven by a general stochastic measure, Statist. Probab. Lett. 146 (2019) 224-230.

[11] K. Shi, Y. Wang, On a stochastic fractional partial differential equation with a fractional noise, Stochastic. 84 (2012) 21-36.

[12] J. Xu, J. C. Liu, Y. Miao, Strong averaging principle for two-time-scale SDEs with nonLipschitz coefficients, J. Math. Anal. Appl. 468 (2018) 116-140.

[13] Y. Xu, J. Duan, W. Xu, An averaging principle for stochastic dynamical systems with Lévy noise, Physica D. 240 (2011) 1395-1401.

[14] Y. Xu, B. Pei, J.-L. Wu, Stochastic averaging principle for differential equations with nonLipschitz coefficients driven by fractional Brownian motion, Stoch. Dyn. 17 (2017) 1750013.

[15] H. G. Yue, Y. Xu, B. Pei, J.-L. Wu, Averaging principles for two-time-scale stochastic differential equations driven by Lévy processes with non-Lipschitz condition. Submitted. 